\title{
EVALUATING THE SAFETY IMPLICATIONS AND BENEFITS OF AN IN-VEHICLE DATA RECORDER TO YOUNG DRIVERS
}

\author{
Tsippy Lotan \\ OR YAROK \\ Ramat Hasharon, Israel \\ E-mail: tsippy@oryarok.org.il \\ Tomer Toledo \\ Transportation Research Institute \\ Technion - Israel Institute of Technology \\ Haifa, Israel \\ E-mail: toledo@technion.ac.il
}

\begin{abstract}
Summary: Young drivers in Israel, as in other parts of the world, are involved in car crashes more than any other age group. Green Light for Life is a new program that seeks to improve the quality of the experience of young drivers during the mandatory accompanied driving period. As part of the efforts to evaluate the effectiveness of this program a novel experiment, which uses information gathered from an in-vehicle data recorder (IVDR) is conducted. The DriveDiagnostics IVDR system, which is used in this study, can identify over 20 different maneuver types in raw measurements and use this information to indicate overall trip safety. Drivers receive feedback through various summary reports, real-time text messages or an in-vehicle display unit. Preliminary validation tests with the system demonstrate promising potential. In the experiment, the DriveDiagnostics system is installed in the primary vehicle driven by the young driver in 120 families. The experiment is designed to test the impact on driving behavior of participation in the program and the type of feedback drivers receive from the system. The data collection part of the experiment is scheduled to run for 8 months for each family.
\end{abstract}

\section{INTRODUCTION}

Young drivers in Israel are involved in car crashes more than any other age group. This observation, which has remained unchanged over the last ten years, is consistent with similar figures worldwide. For example, Williams (2003) quotes higher young driver rates of involvement in car crashes in the US normalized by the number of drivers, miles traveled and population size. This phenomenon has received significant media and political attention in recent years. In Israel, various regulatory changes that affect the driver licensing system were implemented to address this issue. The current licensing system requires that students attend a minimum of 28 practical driving lessons, given by professional driving instructors, before they can apply for a driving test. Young adults can begin taking driving lessons at the age of 16.5 years, but a driving license can only be issued after the age of 17 years. All new drivers, regardless of their age, must be accompanied by an experienced driver whenever they drive for the first three months after obtaining a driving license. 
Various approaches to address the issue of young drivers' involvement in car crashes have been implemented worldwide. Research needs, both in terms of understanding the phenomenon itself and in the evaluation of the effectiveness of the different approaches, are well defined. But, we are still far from achieving a good understanding of the core of the problem and even farther from its solution (Ferguson, 2003; Hedlund et al., 2003; Hedlund \& Compton, 2004). In this paper, we report on a program, titled Green Light for Life, which is aimed at promoting safe driving in young drivers by improving the quality of their accompanied driving experience and at evaluating the impact of this program on young drivers and their families using an in-vehicle data recorder (IVDR) system. Although the tools and experiments described in this paper aim to evaluate the effectiveness of a specific approach, they could be generalized, and so have the potential to ultimately assist in tackling the problem of young drivers' involvement in car crashes.

The rest of this paper is organized as follows: in the next section we describe the Green Light for Life program. The evaluation of the program impact relies on data collected using an IVDR system. We therefore present the details of the specific system being used. Next, we present the experimental design and evaluation methodology that will be used in the research and preliminary results. We conclude with some closing remarks.

\section{GREEN LIGHT FOR LIFE}

OR YAROK, meaning Green Light, is a non-profit organization with the purpose of reducing traffic casualties and improving driving safety in Israel. OR YAROK focuses its activities in four major areas: enforcement, driver education, public motivation, and research. In January 2004, OR YAROK launched a new program, titled Green Light for Life aimed at young drivers and their parents. The program targets the accompanied driving period in which young drivers are required to drive with an experienced driver. The program is intended to reach about 45,000 new young drivers in 2005, and all of the approximately 80,000 new young drivers in Israel by the end of 2006.

The program is conducted as follows. New young drivers are contacted by OR YAROK's representatives and volunteers during their driving learning phase, and a meeting is scheduled at the young driver's home. The meeting, in which both the young driver and parents participate, takes place as close as possible to licensure. In this meeting, which takes approximately an hour, a trained volunteer provides the family with multimedia material, which includes guidance and tools that are designed to help improve the effectiveness of the accompanied driving period. The theme of the meeting and the material in the kit is "Don't be Surprised and Don't Surprise." This theme is interlaced into all guidance materials, which include a CD/DVD that features scenes humoring the difficulties of driving with the parents and stresses the benefits that may be obtained from the parents' experience. The kit also includes written and animated material, which describes difficult driving situations and tips and suggestions on how to avoid these situations and respond to them when they occur. As of April 2005, 6,200 families have joined the program, and about 1,000 young drivers have already completed the guided accompanied driving period.

The Green Light for Life program is followed closely by research to evaluate its impact. So far, the evaluation has mostly been based on solicitation of perceptions and attitudes from 
participants and non-participants through questionnaires and focus groups. Although in its initial stages, the research has already provided some interesting insights and hindsight and highlighted some potential directions for improvement for the program. However, since the research is based on stated preferences approaches, the correlation with observed changes in behavior is lacking. In order to enhance this aspect a unique experiment, which utilizes data from an in-vehicle data recorder, is launched.

\section{IN-VEHICLE DATA RECORDER}

In-vehicle data recorders (IVDR) are on-board devices that record information about the movement, control and performance of the vehicle (NHTSA, 2001; Chidester et al., 2001). Most of the applications of these systems have centered on the car crash event itself (e.g., crash investigations, emergency response, research and development of safety devices). However, the IVDR data may also be used in other avenues, such as prevention and training. The application in this paper demonstrates the usage of an IVDR system to monitor and provide feedback to young drivers about their driving behavior for the purpose of education and training.

The limited empirical evidence reported in the literature indicates that installation of IVDR systems and the fact that drivers know their behavior on the road may be monitored affects driver behavior and safety. For example, Lehmann (1996) reports several case studies in which the installation of IVDR systems in various fleets resulted in reductions of $20-30 \%$ in crash rates, and even more significant reductions in the related costs. While these results are promising, we are not aware of any studies that explain the causes of the safety improvements and therefore how they can be reproduced. For example, it is not clear to what extent these benefits are transferable to private vehicles, where the monitoring itself may not be an important deterrent of unsafe behavior. It is also important to investigate whether or not the safety effects stem from changes in drivers' perceptions and attitudes that would affect driving behavior in the long-run and carry over to trips driven in vehicles that are not equipped with IVDR systems.

DriveDiagnostics is a specialized IVDR system that has been designed for the purpose of monitoring and analyzing driver behavior in normal driving situations, not only in crash events. The overall framework of the DriveDiagnostics system is shown in Figure 1. Using in-vehicle sensors, the system collects information on the movement of the vehicle at a high resolution, which includes the two-dimensional acceleration and speed of the vehicle. Pattern recognition algorithms are applied to the raw measurements to identify and classify over 20 different behaviors and maneuver types in the raw measurements, such as lane changes with and without acceleration, sudden braking, strong accelerations, and so on. The quality of performance of the detected maneuvers is also evaluated using parameters such as the duration and smoothness of maneuvers.

The specific behavioral events that were identified are then synthesized to evaluate the overall trip safety, characterize the driver profile and estimate the associated costs. At the trip level, the observed behavior may be used to make inferences and produce warnings on the driver mental state (e.g., fatigued, distracted). The information that accumulates for each driver may be used to classify drivers along various dimensions, such as levels of experience and aggressiveness. In the current implementation, drivers are classified in three categories (safe, unsafe and dangerous) based on the rate and severity of events they generate. The information is transmitted in real-time 
by wireless networks to an application server, which maintains a database with vehicle-specific and driver-specific trip history and other relevant information, such as crash records, maintenance, fuel costs etc.

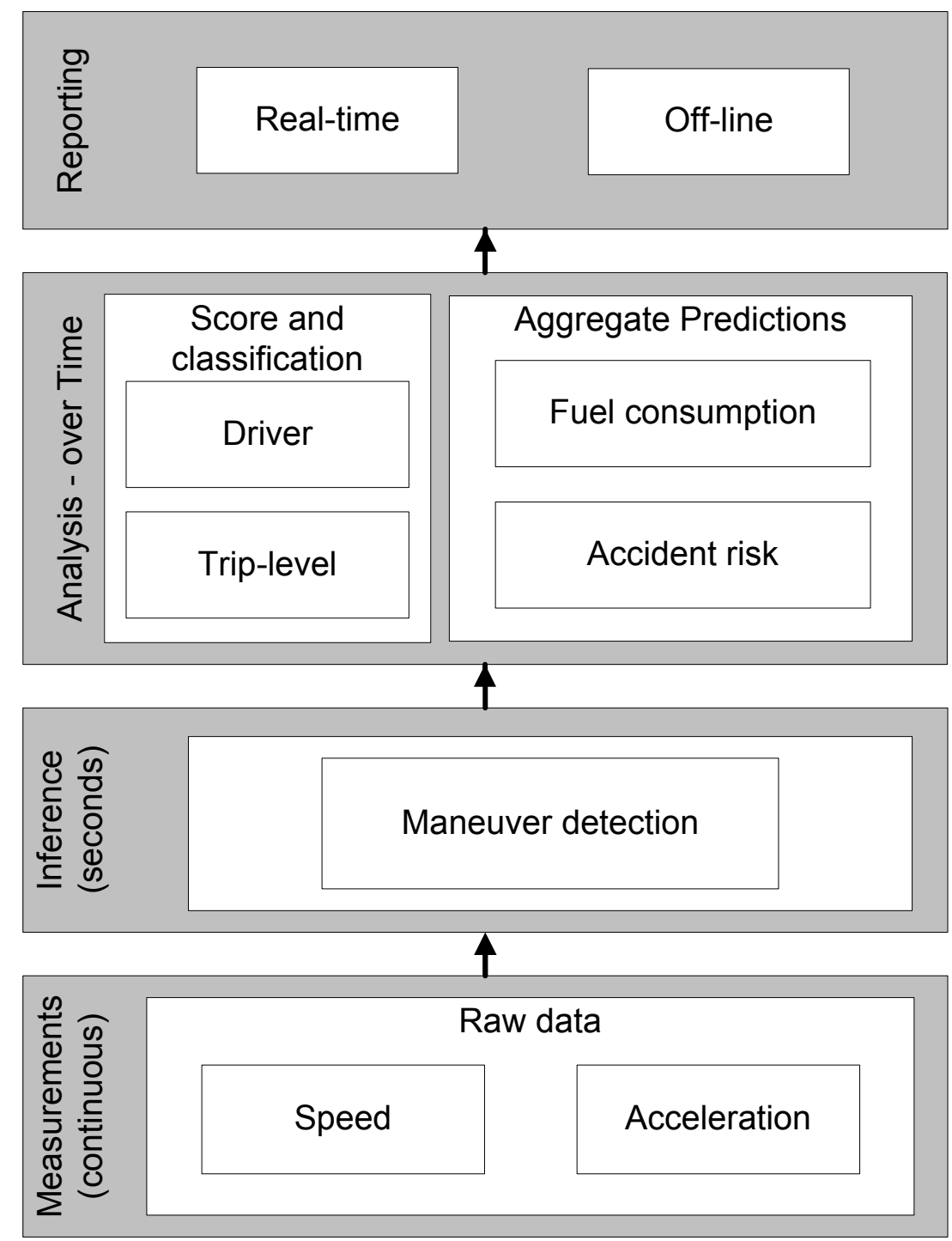

\section{Figure 1. Overall framework of the DriveDiagnostics system}

The information collected in the database may be used both off-line and in real-time. In an offline application, various reports that summarize and compare information at the level of the driver, vehicle, or entire fleet are produced and viewed as printed reports or through a dedicated web site. An example of a monthly driver report is shown in Figure 2. Each square in the summary chart corresponds to a trip, from engine start to turn off. The $\mathrm{X}$ axis indicates the day of the month and the $\mathrm{Y}$ axis indicates the number of trips performed during each day. Trips are color-coded by their safety classification: green, yellow and red for safe, unsafe and dangerous trips, respectively. Real-time feedback, which typically includes warnings on dangerous behavior or significant deviations from the normal driving patterns for the specific driver, can currently be 
provided in two ways: as a text message sent to the driver or to others (e.g., fleet managers, parents of a young driver) or using an in-vehicle display unit.
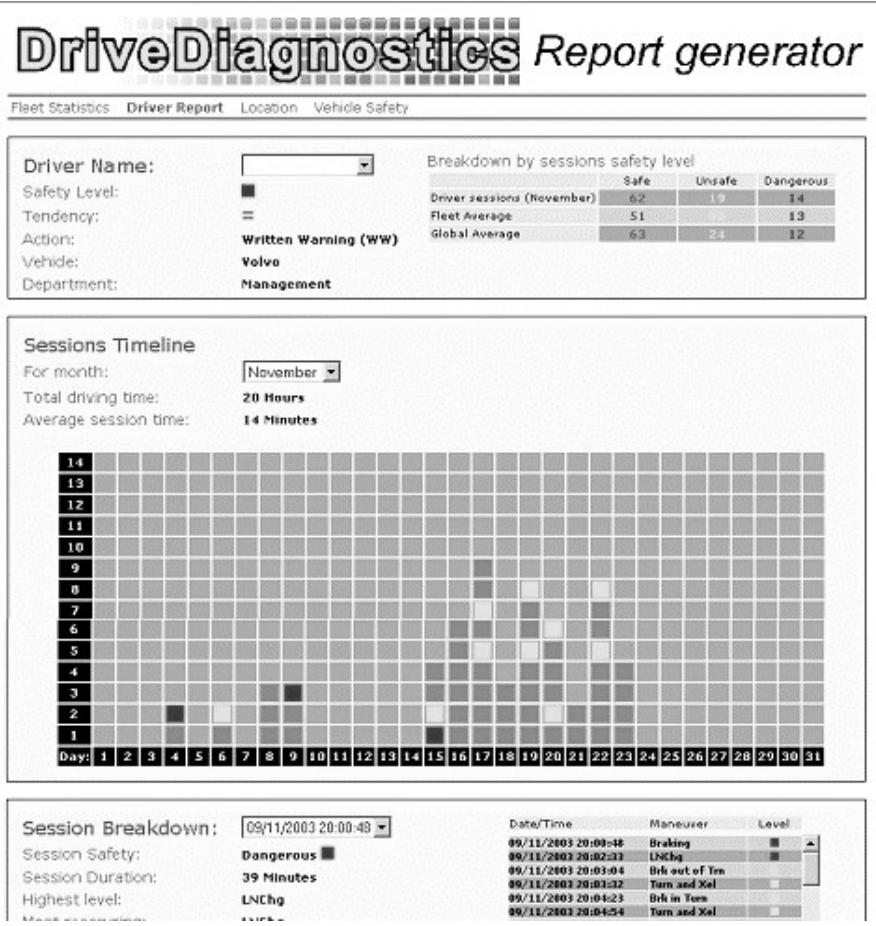

Figure 2. An example of a monthly driver report

In preparation for the research described here, the DriveDiagnostics system was validated as a predictor of traffic safety using data collected from 29 vehicles. Historic accident numbers and costs for the pilot drivers were collected in addition to the information gathered by the IVDR in the initial period before drivers were exposed to the feedback from the system. A strong and significant connection was found between the driver classification and the number and cost of accidents the driver has been involved in. Figure 3 shows the average number of accidents and accident cost per year for drivers in each of the three classes.
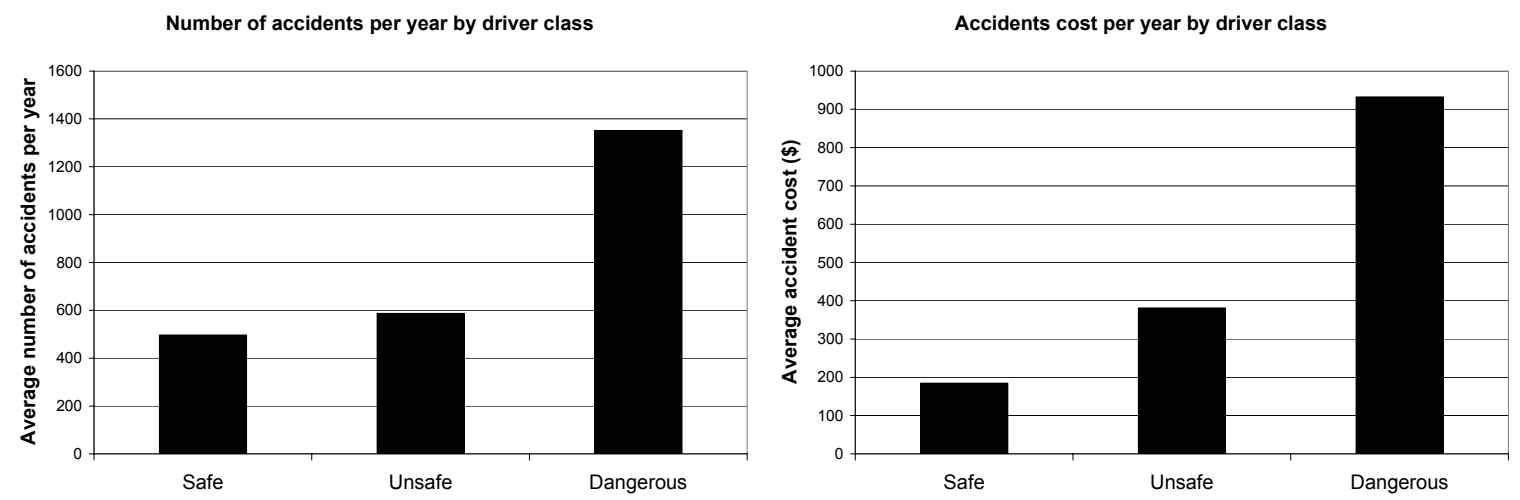

Figure 3. Average number of accidents and accidents costs per year by driver class 


\section{EXPERIMENT}

The DriveDiagnostics system described above is used in an experiment to evaluate the impact of the Green Light for Life program. The DriveDiagnostics system is installed in the primary vehicle driven by the young driver in the 120 families that participate in the experiment. The system tracks all trips made by all family members in the vehicle during the experiment.

The experiment is designed to test the impact of two factors on the driving profile produced by the DriveDiagnostics system as an indicator to driving behavior: participation in the Green Light for Life program and the type of feedback drivers receive from the system. The experiment involves various levels of feedback to participants, ranging from no feedback, through on-line feedback via a secured web site, continuing with text messages sent in real-time to parents when the young driver drives dangerously, and a display unit installed in the car indicating in real-time an event occurrence.

The main issues, which the data collected during the experiment phase will be used as the basis to evaluate, are the following:

- Characterization of the driving profiles of young drivers and their families during the accompanied driving period and thereafter/

- Continuous monitoring of young drivers and identification of specific unsafe maneuvers and behaviors they undertake.

- Monitoring of the transition from the accompanied driving period to the independent one.

- The impact of the various feedback mechanisms on the safety performance of young drivers and their families.

The experiment described here is complemented by questionnaires and focus groups for both the young drivers and their parents. Several indirect benefits and attitudes towards the potential of the DriveDiagnostics system are evaluated. Some examples include its potential to help young drivers cope with peer pressure, the impact on the willingness of parents to give their vehicle to the young drivers, young drivers' attitudes towards aggressive driving and more.

\section{PRELIMINARY RESULTS}

The preliminary results shown here are based on the observations of 2,488 trips made by four pairs of parents and young drivers. Figure 4 summarizes the rates of events recorded for the parents and the young drivers. Overall, young drivers generate 34\% more events per hour compared to their parents. The composition of the types of events parents and young drivers generate also differs. Young drivers generate significantly higher rates of turn-handling events (by 70\%), which indicate unsmooth turns (e.g., braking or accelerating on entry, during or at the exit from turns). Similarly young drivers generate $24 \%$ higher rates of lane-handling events (e.g., abrupt lane changes and bypassing). This is not surprising since these maneuvers require a high level of skill that young drivers may not have mastered yet. On the other hand, young drivers perform about 18\% lower rates of braking and acceleration events compared to their parents. This may be the result of the more cautious behavior of these inexperienced drivers, and their overall lower speed compared to their more self-assured parents. These initial results also seem 
to indicate the importance of the accompanied driving period, which can help young drivers gain important experience and improve their skills.

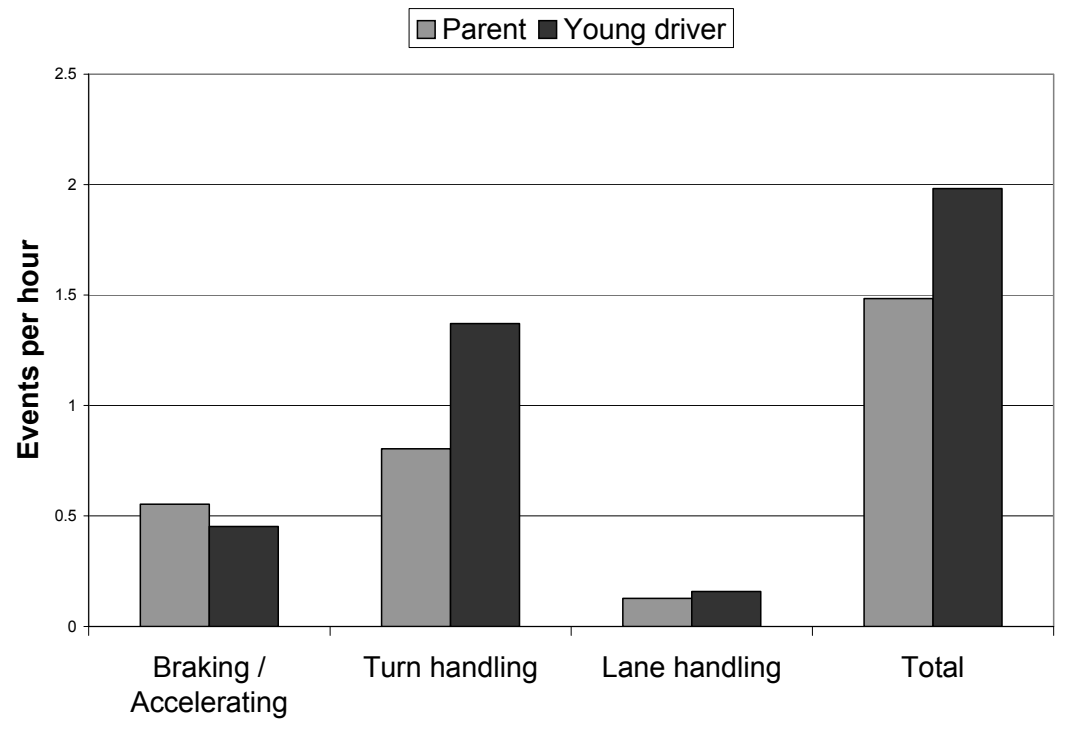

Figure 4. Rates of Events for young drivers and their parents

\section{SUMMARY}

Young drivers in Israel, as in other parts of the world, are involved in car crashes more than any other age group. Various approaches to address this issue have been implemented worldwide.

Green Light for Life is a new program aimed at young drivers and their parents. The program targets the accompanied driving period in which young drivers are required to drive with an experienced driver, and seeks to improve the quality of their accompanied driving experience.

As part of the efforts to evaluate the effectiveness of this program a novel experiment, which uses information gathered from an in-vehicle data recorder (IVDR) system, is conducted. The DriveDiagnostics IVDR system used in this study is specifically designed for the purpose of monitoring and analyzing driver behavior. The system can identify over 20 different maneuver types in the raw measurements, and use this information to indicate on overall trip safety. Drivers receive feedback through various forms of summary reports, or in real-time through SMS messages, or an in-vehicle display unit. Preliminary validation tests with the system demonstrate promising potential in several directions.

In the experiment, the DriveDiagnostics system is installed in the primary vehicle driven by the young driver in 120 families. The experiment is designed to test the impact on driving behavior of the participation in the Green Light for Life program and the type of feedback drivers receive from the system. The data collection part of the experiment is scheduled to run for 8 months for each family. 


\section{REFERENCES}

Chidester, A.C.D., Hinch, J. \& Roston, T.A. (2001). Real world experiences with event data recorders. Proceedings of the 17th International Technical Conference on the Enhanced Safety of Vehicles (ESV), Amsterdam, Holland, paper 247.

Ferguson, S.A. (2003). Other high risk factors for young drivers - How graduated licensing does, doesn't, or could address them. Journal of Safety Research, 34: 71-77.

Hedlund, J. \& Compton, R. (2004). Graduated Driver Licensing Research in 2003 and beyond. Journal of Safety research, 35: 5-11.

Hedlund, J., Shults, R.A. \& Compton, R. (2003). What we know, what we don't know, and what we need to know about Graduated Driver Licensing. Journal of Safety Research, 34: 107-115.

Lehmann, G. (1993). The features of the accident data recorder and its contribution to road safety. Proceedings of the $15^{\text {th }}$ Intemational Technical Conference on Enhanced Safety of Vehicles (ESV), Volume 2: 1565-1568.

NHTSA. (2001). Event data records: summary of findings by the NHTSA EDR working group, Report NHTSA-99-5218.

Williams, A.F. (2003). Teenage drivers: patterns of risk. Journal of Safety Research, 34: 5-15. 